\title{
Risk of Stroke among the Women: Bangladesh Perspective
}

\author{
Md. Azharul Hoque
}

Professor, Department of Clinical Neurology, National Institute of Neurosciences \& Hospital, Dhaka, Bangladesh; Email: azharul59@yahoo.com

Stroke is the third leading cause of death in Bangladesh after coronary heart disease and infectious diseases ${ }^{1}$. The World Health Organization (WHO) ranks mortality due to stroke in Bangladesh as number 84 in the world ${ }^{2}$. In Bangladesh this disease affects people at quite a young age in comparison to people around the world. The mortality rate of stroke increased from $6.0 \%$ in 2006 to $8.57 \%$ in 2011 with an age-adjusted mortality rate of 108.31 per 100000 people in the year of 2011; however, the annual mortality rate per 100,000 people from stroke in Bangladesh has increased by $111.7 \%$ since 1990 , an average of $4.9 \%$ a year ${ }^{2}$. For men, the deadliness of stroke in Bangladesh peaks at age $80+$ and it kills men at the lowest rate at age 5-9, in contrast women are killed at the highest rate from stroke in Bangladesh at age $80+$ which was least deadly to women at age 1-4 years ${ }^{3}$. The peak mortality rate for women was higher than that of men which was 4762.3 and 4437.4 deaths per 100,000 deaths in the years of 2013 respectively $^{2}$. The crude death rate per 1000 people in Bangladesh is reported at $5 \cdot 8 \% \%^{3}$. However, stroke in women is poorly reported in South Asia, despite being a leading cause of death in females above the age of 60 years in this region ${ }^{4}$. Hospital-based studies have suggested a lower prevalence of stroke in women than in men, although this figure could be skewed by the fact that women with stroke or cardiac arrest in this part of the world are less likely to be taken to hospital than are men with these conditions.

World Stroke Day observes on October 29 in every year. It is a part of the global effort to raise awareness of stroke for women. It has been already mentioned that women run a higher risk of a stroke globally and are more likely than men to die. In addition, one in five women globally will experience a stroke in their lifetime, compared to one in six men ${ }^{2}$. Other than their longer life expectancy, women have an increased burden of major stroke risk factors including hypertension, irregular heartbeat, diabetes, depression and obesity ${ }^{1}$. Furthermore, specific risk factors and settings in women include pregnancy, the post-partum period and some hormonal replacement therapies. Some kinds of stroke, such as aneurysmal subarachnoid haemorrhage and cerebral venous thrombosis, are more common in women6. For all these reasons, a greater awareness and focus on women and stroke is a global priority for the WSO. Additionally, young adults and people nearing forty who have diabetes, hypertension, and history of heart disease in the family, or are a smoker (even occasionally) are more prone to a stroke at a younger age.

In Bangladesh common risk factors of stroke are age, sex, family history, hypertension, diabetes, ischemic heart disease, smoking, obesity, dyslipidaemia, alcoholism, use of oral contraceptive pill, lack of fresh fruit consumption and so one. Majority of the stroke events occurred in Bangladesh after the age of forty $(82.3 \%)$ and the ischemic stroke $(72 \%)$ is the most common; however, part from non modifiable risk factors hypertension was the most common modifiable risk factor found in stroke patients $(57.6 \%)$ followed by smoking (44.6\%), tobacco use $(24.3 \%)$, OCP use in female $(40 \%$ of female stroke), diabetes (23\%), ischemic heart disease (17.1\%), obesity $(10.6 \%)$ and dyslipidaemia $(5.3 \%)^{1}$. Lack of fresh fruit consumption and alcoholism has also some role of causation of stroke. Stroke is common after the age of forty? Ischemic events are commonest type of stroke. Hypertension, smoking, tobacco use, diabetes and ischemic heart disease are five most common risk factors of stroke. Two studies looking at arterial ischaemic stroke and CVT in young women aged 15 to 45 years from eight Asian countries found that large-vessel thrombosis (24\%), CVT (21\%) and cardioembolism (19\%) were the most common mechanisms of stroke in this population'. A high proportion of these strokes were pregnancy-related. This region probably represents the area of highest prevalence for pregnancy-related and postpartum stroke ${ }^{3}$.

Stroke is preventable and this information should reach the entire country, even across the villages. Outlining 
the common stroke risk factors in the hospital settings, may help the physicians and care givers in managing this disabling disease properly.

[Journal of National Institute of Neurosciences Bangladesh 2016; 2(1): 1-2]

\section{References}

1. Ekenze OS1, Onwuekwe IO, Ezeala Adikaibe BA. Profile of neurological admissions at the University of Nigeria Teaching Hospital Enugu. Niger J Med. 2010;19(4):419-22

2. World Health Organization. Neurological disorders: public health challenges. World Health Organization; 2006.; Viewed on: 9 September 2016; Web Address: http://www.who.int/ mental_health/ neurology/neurodiso/en/
3. Murray CJL, Lopez AD, eds. The global burden of disease: a comprehensive assessment of mortality and disability from diseases, injuries and risk factors in 1990 and projected to 2020. Cambridge, MA, Harvard School of Public Health on behalf of the World Health Organization and the World Bank, 1996 (Global Burden of Disease and Injury Series, Vol. I)

4. Philip-Ephraim EE1, Eyong KI, Chinenye S, William UE, Ephraim RP. The burden of inpatient neurologic disease in a tropical African hospital. Can J Neurol Sci. 2013 Jul;40(4):576-9

5. Eze CO, Kalu UA. Pattern of neurological admissions in the tropics: experience at Abakaliki South-Eastern Nigeria. Niger $\mathrm{J}$ Med 2014;23(4):302-5

6. Chowdhury RN, Hasan ATMH, Rahman YU, Khan SI, Hussain AR, Ahsan S. Pattern of neurological disease seen among patients admitted in tertiary care hospital. BMC Research Notes 2014;7:202

7. Bhopal R, Rahemtulla T, Sheikh A: Persistent high stroke mortality in Bangladeshi populations. BMJ. 2005, 331: 1096-1097 